Check for updates

Cite this: RSC Adv., 2018, 8, 37765

Received 21st September 2018 Accepted 3rd November 2018

DOI: $10.1039 / \mathrm{c} 8 \mathrm{ra07875k}$

rsc.li/rsc-advances

\section{Facile preparation of degradable multi-arm-star- branched waterborne polyurethane with bio-based tannic acid}

\begin{abstract}
Shun Luo, Kai Yang, Zhen Zhong, Xiaojian Wu and Tianbin Ren (iD*
In this research, biodegradable multi-arm-star-branched waterborne polyurethanes (MWPUs) were prepared by incorporation of bio-based material (tannic acid, TA) in the structure of waterborne polyurethanes. The prepared MWPUs were characterized by UV-vis spectrometry and FT-IR spectrometry, confirming the presence of TA in MWPUs. The results of DSC and TGA demonstrated that the incorporation of TA remarkably enhanced the thermal stability of MWPUs. The mechanical strength test indicated that the Young's modulus and tensile strength of the waterborne polyurethanes after incorporation of TA were significantly improved due to the increase of structural rigidity, hydrogen bonding and the molecular interactions of the TA-based MWPU chains. In addition, the synthesized TA-based MWPUs also exhibited excellent antioxidation capacity and outstanding biodegradation property. Given these excellent properties and the sustainability of TA, the developed TA-based MWPUs exhibited great potential in a wide range of practical applications.
\end{abstract}

\section{Introduction}

Eco-friendly waterborne polyurethanes (WPUs) have gained increasing attention in a wide range of applications including coatings, adhesives, elastomers and biomedicine due to their good flexibility, non-toxicity and nonflammability as well as their reduced volatile organic compounds (VOCs) release compared with their solventbased counterparts. ${ }^{1-3}$

Recently, bio-based raw materials such as castor oil and soybean oil have been exploited as components to synthesize WPUs, providing an effective and efficient approach for developing bio-based WPUs. ${ }^{4-6}$ The biological polyurethane material is non-toxic, biodegradable and biocompatible, which is very promising in commercial applications. The degradation properties of biodegradable materials as medical materials are of interest, as a long-term material implanted in the body is generally not expected to degrade. But when used as similar tissue engineering scaffolds, medical adhesives and drug sustained-release materials, it is hoped that they can be gradually degraded in the body. Besides, biodegradable multi-arm-star-branched waterborne polyurethane can be used to make biodegradable condoms. ${ }^{7-10}$

Institute of Nano and Biopolymeric Materials, School of Materials Science and Engineering, Key Laboratory of Advanced Civil Engineering Materials, Ministry of Education, Tongji University, 4800 Caoan Road, Shanghai 201804, China. E-mail: rentianbin008@163.com; Fax: +86-21-33515906; Tel: +86-21-33515906
In this research, biodegradable multi-arm-star-branched waterborne polyurethanes (MWPUs) were prepared by incorporation of bio-based material (tannic acid, TA) in the structure of waterborne polyurethanes. The structure of the prepared MWPUs was characterized with UV-vis spectrometer and FT-IR spectrometer. The effects of incorporation of TA on the thermal stability and mechanical properties of WPUs were investigated. Furthermore, the antioxidation capacity of TA-based MWPUs was tested via DPPH and hydroxyl radical scavenging assay. Finally, the biodegradation behaviour of TA-based MWPUs in simulated body fluid and natural soil was discussed.

\section{Experimental}

\section{Materials}

Bis(hydroxymethyl) propionic acid (DMPA) was purchased from Shanghai Demand Chemical Co., Ltd, China. Poly (tetramethylene ether glycol) (PTMG, $\mathrm{Mn}=2000$ ) and isophorone diisocyanate (IPDI) were obtained from Jining Baichuan Chemical Co., Ltd, China. L-Ascorbic acid and di(phenyl)(2,4,6-trinitrophenyl) iminoazanium (DPPH) were obtained from Sigma Aldrich (St. Louis, MO, USA). Dibutyltin dilaurate and stannous octoate were provided by Jiangsu Yoke Technology Co., Ltd, China. Triethylamine (TEA), diethylene glycol (DEG), tannic acid (TA), tetrahydrofuran (THF), ethanol, hydrogen peroxide, 2-hydroxybenzoic acid, ferrous sulfate, sodium chloride, potassium chloride, sodium dihydrogen phosphate and potassium dihydrogen phosphate were 
obtained from Sinopharm Chemical Reagent Shanghai Co., Ltd., China and used as received.

\section{Preparation of TA-based MWPUs and films}

The synthesis process of TA-based MWPUs is shown in Scheme 1. A typical procedure is as follows: PTMG-2000, TA and DMPA were preprocessed to remove the moisture before using via drying them in a vacuum oven at $110^{\circ} \mathrm{C}, 75^{\circ} \mathrm{C}$ and $75^{\circ} \mathrm{C}$ for $2 \mathrm{~h}$, respectively. Then, PTMG-2000 (100 g, $0.05 \mathrm{~mol})$ was placed in a $50 \mathrm{ml}$ four-neck round-bottom flask under $65{ }^{\circ} \mathrm{C}$, followed by addition of IPDI ( $33 \mathrm{~g}, 0.15 \mathrm{~mol})$. Afterwards, the mixture was heated to $80{ }^{\circ} \mathrm{C}$ and kept for $2 \mathrm{~h}$ to prepare the -NCO-ended polyurethane prepolymer. DMPA $(7.98 \mathrm{~g}, 0.06 \mathrm{~mol})$, DEG, dibutyltin dilaurate and stannous octoate were then added for further reaction of $4 \mathrm{~h}$ to get the polyurethane prepolymer with $-\mathrm{COOH}$ groups. The mixture was cooled to $25^{\circ} \mathrm{C}$, and then TA solution dissolved in THF was added, followed by keeping the reaction at $60{ }^{\circ} \mathrm{C}$ for $5 \mathrm{~h}$. After that, the mixture was placed in a water bath until the temperature dropped to $25^{\circ} \mathrm{C}$, and was then neutralized by slowly adding TEA ( $6.02 \mathrm{~g}, 0.06 \mathrm{~mol})$ under stirring (175-200 rpm) in $45 \mathrm{~min}$. Subsequently, the polymer mixture was vigorously stirred (1400-1500 rpm) for $30 \mathrm{~min}$ and emulsified with a certain amount of deionized water to obtain a WPU dispersion. Finally, the WPU solution with solid content of $35 \%$ was obtained by removal of THF via vacuum evaporation. A series of WPUs (WPU0, MWPU1, MWPU2, MWPU3) were prepared via adjusting the ratios of TA (Table 1).

The polyurethane films were prepared by pouring WPUs and MWPUs dispersions into PTFE module, and then drying at room temperature for $24 \mathrm{~h}$. Then as-prepared films with thickness of $0.5 \mathrm{~mm}$ was further dried in a vacuum oven to a constant weight at $60{ }^{\circ} \mathrm{C}$ and were then stored in a desiccator prior to testing and characterization.

\section{Free radical scavenging assay}

DPPH free radical scavenging assay. The mechanism of DPPH radical scavenging assay was shown in Fig. 1. Typically, $\mathrm{DPPH}^{-}$is an available organic nitrogen radical in ethanol solution and has strong absorption maximum at $517 \mathrm{~nm}$. $\mathrm{DPPH}^{\cdot}$ can accept hydrogen from an antioxidant to form DPPH2, which results in the colour of solution turning from purple to yellow and the decrease of UV absorption at $517 \mathrm{~nm}$. Therefore, the antioxidant capacity can be easily evaluated by measurement of the UV absorption at $517 \mathrm{~nm}$ of $\mathrm{DPPH}^{\circ}$ solution.

In our study, to test the radical scavenging activity, $1 \mathrm{ml}$ of $0.1 \mathrm{M}$ DPPH (in 50\% ethanol solution) was mixed with $1 \mathrm{ml}$ of tannic acid-based hyperbranched WPUs solution $(0.2-2.0 \mathrm{mg}$ $\mathrm{ml}^{-1}$ in double distilled water). The reaction mixture was incubated at room temperature for 20 min under dark conditions. Then the absorbance was measured at $517 \mathrm{~nm}$, and the scavenging activity of tannic acid-based hyperbranched WPUs was measured by using the following eqn (1),

$$
\text { Scavenging efficiency }(\%)=1-\frac{A_{1}}{A_{0}} \times 100 \%
$$

where $A_{1}$ is the absorbance of DPPH solution with the presence of samples and $A_{0}$ is the absorbance of the control. For the control, a same volume of DI water was added into the test tube instead of polymeric solution. In addition, L-ascorbic acid was used as a positive control. ${ }^{11-13}$

Hydroxyl free radical scavenging assay. To measure the hydroxyl radical scavenging, the prepared multi-arm-starbranched WPUs was dissolved in deionized water with concentration of $0.2-2.0 \mathrm{mg} \mathrm{ml}^{-1} 2 \mathrm{ml}$ of polymeric solution was taken out and added into the test tube. Then, ferrous sulfated aqueous solution $(0.1 \mathrm{M})$ and salicylic acid solution $(0.1$ $\mathrm{M})$ in ethanol were added into the test tube, followed by adding $\mathrm{H}_{2} \mathrm{O}_{2}(0.1 \mathrm{M}, 2 \mathrm{ml})$. The mixture was shaken well and incubated in $37^{\circ} \mathrm{C}$ water bath for $30 \mathrm{~min}$. The absorbance was measured at $510 \mathrm{~nm}$, and the scavenging ability for hydroxyl radicals was calculated by using the following eqn (2),

$$
\text { Scavenging efficiency }(\%)=1-\frac{A_{\mathrm{a}}}{A_{\mathrm{b}}} \times 100 \%
$$

where $A_{\mathrm{a}}$ is the absorbance of the samples and $A_{2}$ is the absorbance of the control. For the control, a same volume of DI water was added into the test tube instead of polymeric solution. In addition, L-ascorbic acid was used as a positive control. $^{14-16}$

\section{Degradation behaviour of MWPUs in the simulated body fluid}

Before testing degradation behaviour of WPU and multi-armstar-branched WPU films in the simulated body fluid, the film coupons with dimension of $10 \mathrm{~mm} \times 10 \mathrm{~mm} \times 1 \mathrm{~mm}$ were dried in a vacuum oven to a constant weight at $50{ }^{\circ} \mathrm{C}$ and weighed via digital balance $\left(W_{0}\right)$. Then the coupons were immersed into a glass vial containing $10 \mathrm{ml}$ of the PBS simulated body fluid $(\mathrm{pH}=7.4)$ which was prepared by dissolving $0.8 \mathrm{~g}$ of $\mathrm{NaCl}, 0.2 \mathrm{~g}$ of KCl, $1.44 \mathrm{~g}$ of $\mathrm{NaHPO}_{4}$, and $0.24 \mathrm{~g}$ of $\mathrm{KH}_{2} \mathrm{PO}_{4}$ in $1 \mathrm{~L}$ of DI water, followed by incubation at $37^{\circ} \mathrm{C}$. The PBS simulated body fluid was replaced once a day, and the coupons were taken out at predetermined time intervals, washed with DI water for three times and dried in the vacuum oven at $45{ }^{\circ} \mathrm{C}$. The weight of dried coupons $\left(W_{\mathrm{t}}\right)$ were then measured. The weight loss ratio was calculated using the eqn (3),

$$
\text { Weight loss ratio }(\%)=\frac{W_{0}-W_{\mathrm{t}}}{W_{0}} \times 100 \%
$$

where $W_{0}$ and $W_{\mathrm{t}}$ are the weights of the samples before and after degradation..$^{7,18}$

\section{Biodegradation study of multi-arm-star-branched WPU in soil}

The biodegradation behaviour of multi-arm-star-branched WPU in soil was further investigated following a previously reported protocol. Typically, the soil taken from the lawn located in the Department of Material Science of Tongji University was dried under sunlight for three days. Then the soil was shattered, and the grain of sand was removed from soil. The treated soil was placed in a $250 \mathrm{ml}$ beaker, and $100 \mathrm{ml}$ of DI water was then 
<smiles>CCCCCCC(C)(C)OCC(C)(C)O</smiles>

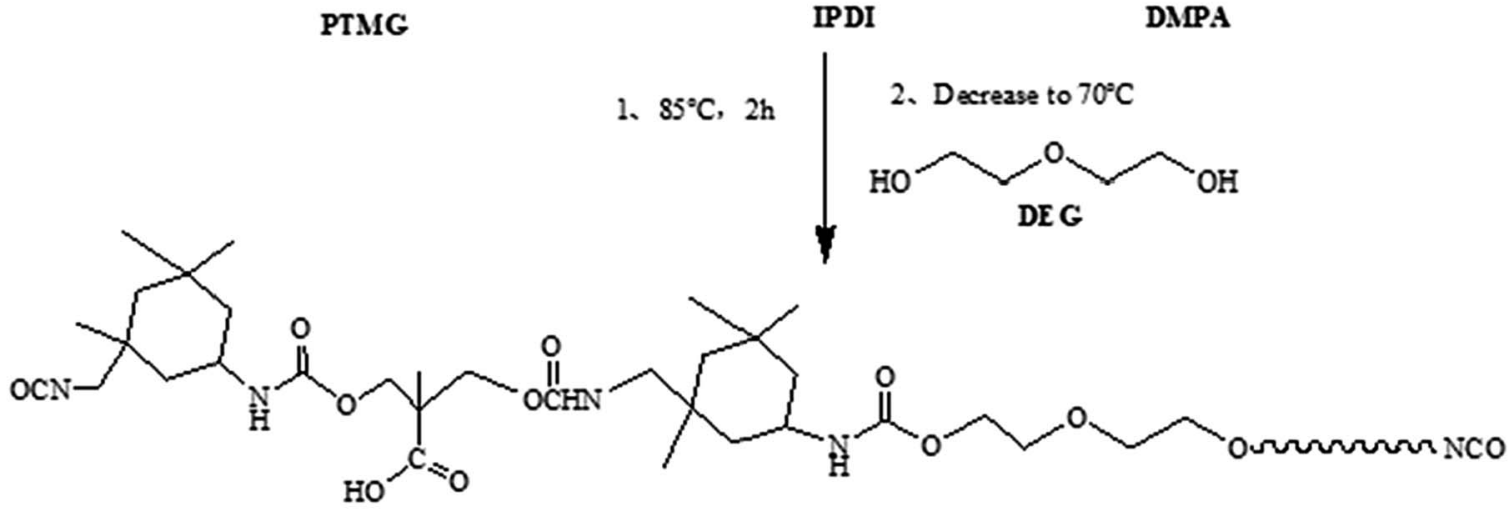

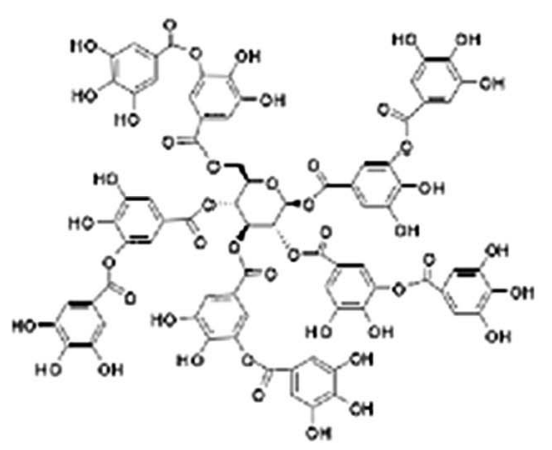

NCO-terminated Prepolymer

TA

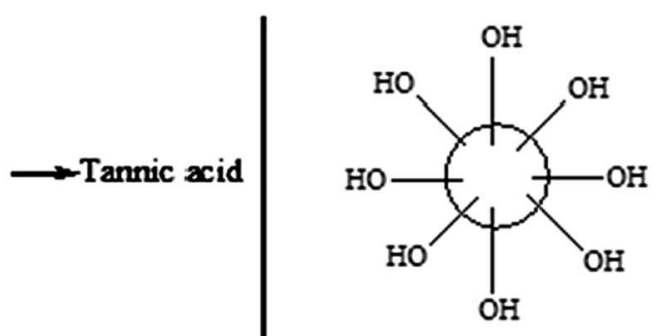

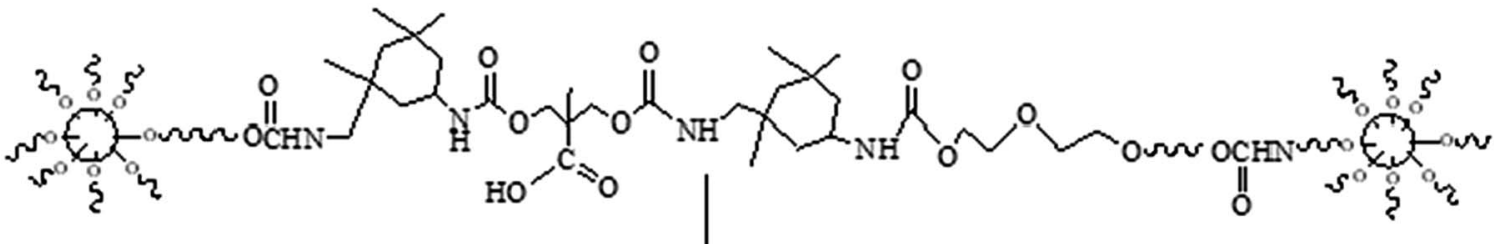

TEA

Stirring

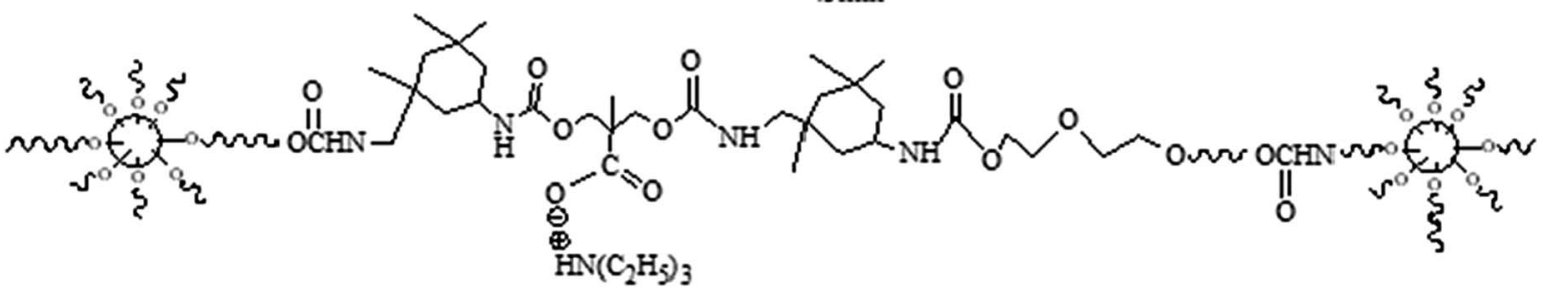

Scheme 1 Synthesis of TA-based MWPUs.

added into the beaker. Before introducing the film samples into the pasty soils, each sample was cut into pieces of dimension of $10 \mathrm{~mm} \times 10 \mathrm{~mm} \times 1 \mathrm{~mm}$ and dried in a vacuum oven to a constant weight $\left(W_{\mathrm{i}}\right)$ at $50{ }^{\circ} \mathrm{C}$. The beaker containing the film samples were buried in a soil bed at a depth of around $15-20 \mathrm{~cm}$ from the top of the ground surface. The biodegradation experiments were carried out under ambient conditions with regular addition of water (except in rainy days). Samples were taken out carefully from each beaker after 30 days, 60 days, 90 days and 120 days, respectively, and washed repeatedly with water to remove the adhered soil from the surface of the films. Then the samples were dried in a vacuum oven until a constant weight was obtained. Finally, the weight of samples was measured $\left(W_{t}\right)$. The weight loss ratio was calculated using the eqn (4), 
Table 1 Composition of reactants for synthesis of WPUs and TAbased MWPUs

\begin{tabular}{lllll}
\hline Materials & WPU0 & MWPU1 & MWPU2 & MWPU3 \\
\hline PTMG (mol) & 1.00 & 1.00 & 1.00 & 1.00 \\
IPDI (mol) & 3.00 & 3.00 & 3.00 & 3.00 \\
DMPA (mol) & 1.20 & 1.20 & 1.20 & 1.20 \\
DEG (mol) & 0.30 & 0.23 & 0.15 & 0.18 \\
TA (mol) & 0 & 0.05 & 0.10 & 0.15 \\
TEA (mol) & 1.20 & 1.20 & 1.20 & 1.20 \\
NCO/OH (ratio) & 1.20 & 1.20 & 1.20 & 1.20 \\
& & & & \\
\hline
\end{tabular}

$$
\text { Weight loss ratio(\%) }=\frac{W_{\mathrm{i}}-W_{\mathrm{t}}}{W_{\mathrm{i}}} \times 100 \%
$$

where $W_{\mathrm{i}}$ and $W_{\mathrm{t}}$ are the weights of the samples before and after degradation. ${ }^{19-22}$

\section{Characterization}

The IR spectra of the prepared multi-arm-star-branched WPUs were obtained using a Nicolet 6700 FT-IR Spectrometer (Thermo Scientific), and the structure of the film samples before and after degradation was measured under the attenuated total reflection (ATR) mode. To evaluate the relative molecular weight and its distribution of the synthesized multi-arm-star-branched WPUs, gel permeation chromatography (GPC) spectrum was measured in THF with a concentration of $2 \mathrm{mg} \mathrm{ml}^{-1}$. The flow rate and temperature were set at $1.0 \mathrm{ml} \mathrm{min}{ }^{-1}$ and $40{ }^{\circ} \mathrm{C}$, respectively. The intrinsic viscosity $\left[\eta_{\mathrm{r}}\right]$ of the synthesized multiarm-star-branched WPUs solutions was determined at $20{ }^{\circ} \mathrm{C}$ with a Bohlin Rotational Rheometer (Malvern Instrument, UK) equipped with cone-and-plate geometry ( $40 \mathrm{~mm}$ diameter, cone angle $4^{\circ}$ ). The rotate speed was 300 radians per second. UV-vis absorption spectra of the WPUs and multi-arm-star-branched WPUs samples were recorded on a HITACHI U-1800 spectrophotometer at room temperature. The concentration of the WPUs and multi-arm-star-branched WPUs solutions was $0.1 \mathrm{mg}$ $\mathrm{ml}^{-1}$. The surface morphology of the film samples prepared with WPUs and multi-arm-star-branched WPUs was observed using a scanning electron microscopy (SEM, FEI, USA). All samples were coated with $10 \mathrm{~nm}$ thick platinum (Pt) before SEM observation. To study the thermal stabilities of the WPUs and multi-arm-star-branched WPUs, thermogravimetric analysis (TGA) was performed on TA Instruments Q500 (New Castle, DE,

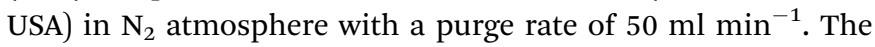
data was recorded from 20 to $800{ }^{\circ} \mathrm{C}$ with a scanning rate of
$20{ }^{\circ} \mathrm{C} \min ^{-1}$. Besides, differential scanning calorimetry (DSC) was also carried out on a TA Instruments DSC-Q20 from -80 to $200{ }^{\circ} \mathrm{C}$ with a heating/cooling rate of $10{ }^{\circ} \mathrm{C} \mathrm{min}^{-1}$ in nitrogen atmosphere. To study the mechanical properties of the samples, the tensile strength, Young's modulus and elongation at break were studied by using a tensile tester (INSTRON5565, USA) at

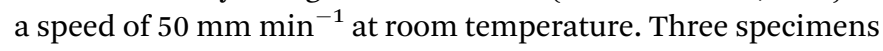
were tested and averaged for each sample. The specimen was molded into $45 \mathrm{~mm}$ long, $8 \mathrm{~mm}$ wide and $0.2 \mathrm{~mm}$ thick.

\section{Results and discussion}

\section{Characterization of MWPUs with TA}

As shown in Scheme 1, there are five branches in the structure of TA, which are generating from a pyranose heterocycle, 25 hydroxyl groups, and 10 ester linkages. Therefore, in our study, bio-based tannic acid was used as an alternative to polyol to synthesize the multi-arm-star-branched WPUs. To confirm the presence of TA in the synthesized MWPU1, MWPU2 and MWPU3, the UV-vis absorption spectroscopy was used to track the characteristic absorbance peak of the TA at around $280 \mathrm{~nm}$. As shown in Fig. 2a, the prominent absorption peaks were observed for MWPU1, MWPU2 and MWPU3, and the intensity of peaks of the synthesized WPUs exhibited an obvious increase ranging from MWPU1 to MWPU3, indicating the higher TA content in the structure of MWPU3 compared with MWPU1 and MWPU2.

FT-IR spectroscopy was further employed to assess the chemical structures of synthesized MWPUs with different TA content. As shown in Fig. 2b, for the spectrum of MWPU1, the absorption peaks focused at $3422 \mathrm{~cm}^{-1}, 1705 \mathrm{~cm}^{-1}$ and $1556 \mathrm{~cm}^{-1}$ are mainly attributed to the symmetric stretching vibration of $-\mathrm{N}-\mathrm{H}$ bond, $-\mathrm{C}=\mathrm{O}$ group and the bending vibration of $-\mathrm{N}-\mathrm{H}$ bond, respectively, indicating the successful formation of carbamate in MWPU1. The peaks observed at $2940 \mathrm{~cm}^{-1}, 2860 \mathrm{~cm}^{-1}, 1105 \mathrm{~cm}^{-1}$ and $1254 \mathrm{~cm}^{-1}$ are ascribed to the symmetric stretching vibration of $-\mathrm{CH}_{2}$ group, the asymmetric stretching vibration of $-\mathrm{CH}_{3}$ group, the symmetric blending vibration of $\mathrm{C}-\mathrm{O}-\mathrm{C}$ bond and the stretching vibration of $-\mathrm{C}-\mathrm{N}$ bond. In addition, the peak positions of $-\mathrm{C}-\mathrm{N}$ bonds in the spectra of MWPU2 and MWPU3 exhibited an obvious blue shift, which is due to the hydrogen bonds formed between the catechol groups of TA and $-\mathrm{C}-\mathrm{N}$ bond and $-\mathrm{C}=\mathrm{O}$ group of carbamates, and the number of hydrogen bond increases with the increase of TA content in MWPU2 and MWPU3. This result
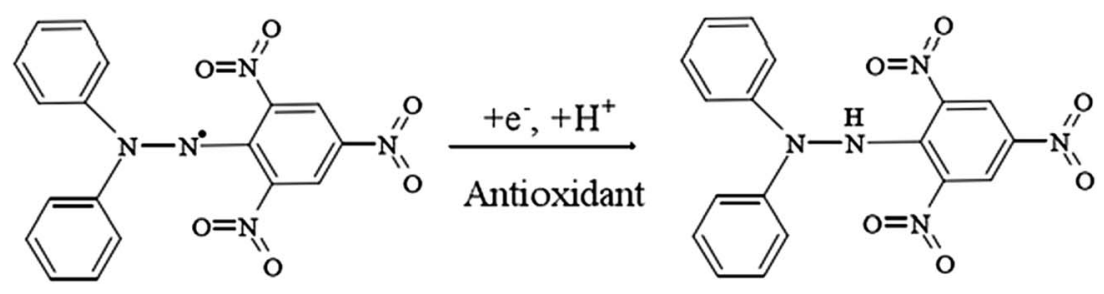

Fig. 1 Mechanism of DPPH radical scavenging assay. 

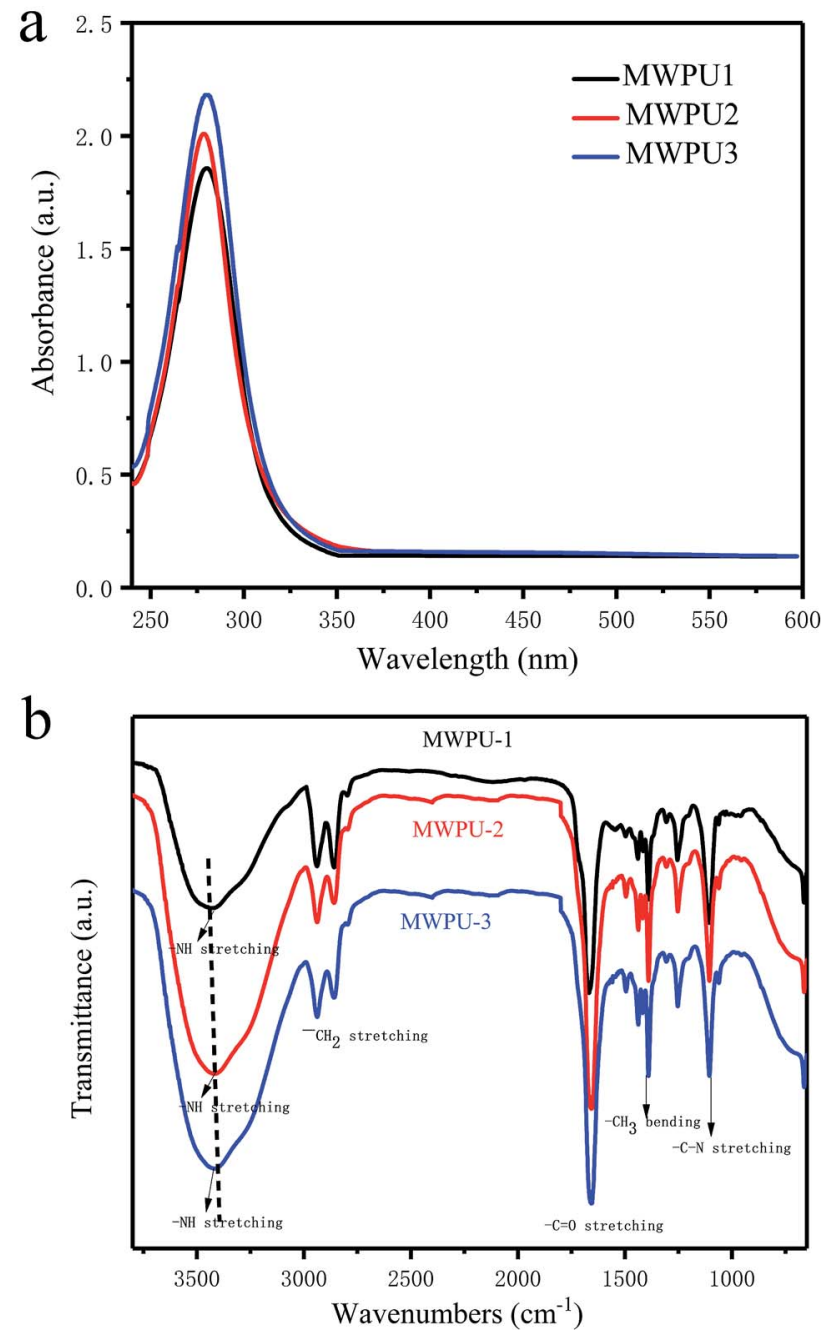

Fig. 2 UV-vis spectra (a) and FT-IR spectra (b) of synthesized MWPU1, MWPU2 and MWPU3.

further confirmed the presence of $\mathrm{TA}$ in the synthesized MWPU1, MWPU2 and MWPU3.

Then the molecular weight and distribution of the synthesized MWPU1, MWPU2 and MWPU3 were measured via GPC and the results presented in Table 2 revealed that the weight average molecular weight $\left(M_{\mathrm{w}}\right)$ of the obtained MWPU3 exhibited a significant increase to $47200 \mathrm{Da}$ from $33100 \mathrm{Da}$ of MWPU1 with the increase of the addition amount of TA in the reaction system. The polydispersity index (PDI) of the obtained MWPU1, MWPU2 and MWPU3 was measured to be 1.40, 1.34, and 1.34, respectively, indicating that all MWPUs have narrow molecular weight distribution and the TA content in the MWPUs did not affect the molecular weight distribution significantly. Thus, the molecular weights of the MWPUs can be well controlled via adjusting the addition amount of TA in the synthesis process, without affecting the molecular weight distribution. The intrinsic viscosities of the obtained MWPU1, MWPU2 and MWPU3 solution were determined with a rotational rheometer. Compared with the reported WPUs, MWPUs prepared with TA exhibited clearly reduced intrinsic viscosities
Table 2 Weight average $\left(M_{w}\right)$ and number average $\left(M_{n}\right)$ molecular weight, PDI and solution viscosity of synthesized MWPUs

\begin{tabular}{llll}
\hline Parameter & MWPU1 & MWPU2 & MWPU3 \\
\hline$M_{\mathrm{w}}$ & 33100 & 45000 & 47200 \\
$M_{\mathrm{n}}$ & 23600 & 33600 & 35200 \\
PDI & 1.40 & 1.34 & 1.34 \\
Viscosity $\left(\mathrm{ml} \mathrm{g}^{-1}\right)$ & 0.441 & 0.349 & 0.321
\end{tabular}

(0.441, 0.349 and $0.321 \mathrm{mg} \mathrm{L}^{-1}$ for MWPU1, MWPU2 and MWPU3, respectively), which is due to the very compact threedimensional structures of MWPU1, MWPU2 and MWPU3 that reduces the chain interactions significantly. This result is also generally consistent with the previously reported results of other hyperbranched polymers.

\section{The influences of TA on the thermal properties of the synthesized MWPUs}

To evaluate the influences of TA on thermal properties of MWPUs, the samples of WPU0 and MWPUs were analysed by DCS and TGA. As shown in Fig. 3, an obvious absorption peak at $15{ }^{\circ} \mathrm{C}$ appeared for the sample of WPU0 due to the melting of the soft segment, indicating a certain degree of microcrystallization. However, the other MWPUs samples did not have any melting absorption peaks, indicating that the introduction of rigid tannic acid disturbed the crystallization region of the pristine waterborne polyurethane.

As shown in Fig. 4, the synthesized WPU0 and MWPUs exhibited three-stage thermal degradation process: (i) at the first stage, the decomposition of some thermolabile urethane bonds and ester bonds occurred around $250^{\circ} \mathrm{C}$; (ii) the second stage was corresponding to the decomposition of the aromatic rings from tannic acid (TA) around $300{ }^{\circ} \mathrm{C}$; (iii) the third stage was around $420^{\circ} \mathrm{C}$, involving the decomposition of the soft segment on the polyurethane backbone. Furthermore, the temperatures at different weight loss ratios for the samples of WPU0 and MWPUs were listed in Table 3. It could be seen that as the content of TA increases, the thermal decomposition temperature of the

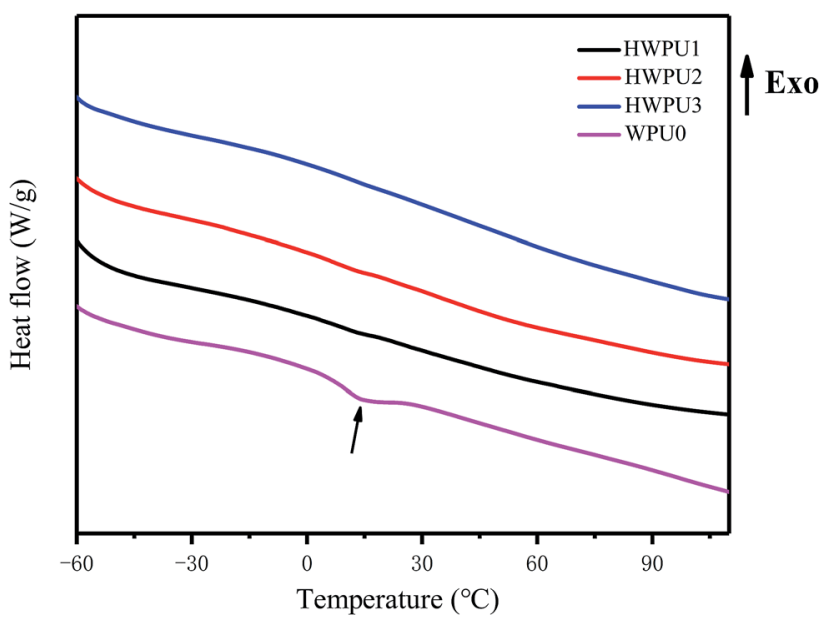

Fig. 3 DSC curves of synthesized WPUO and MWPUs. 


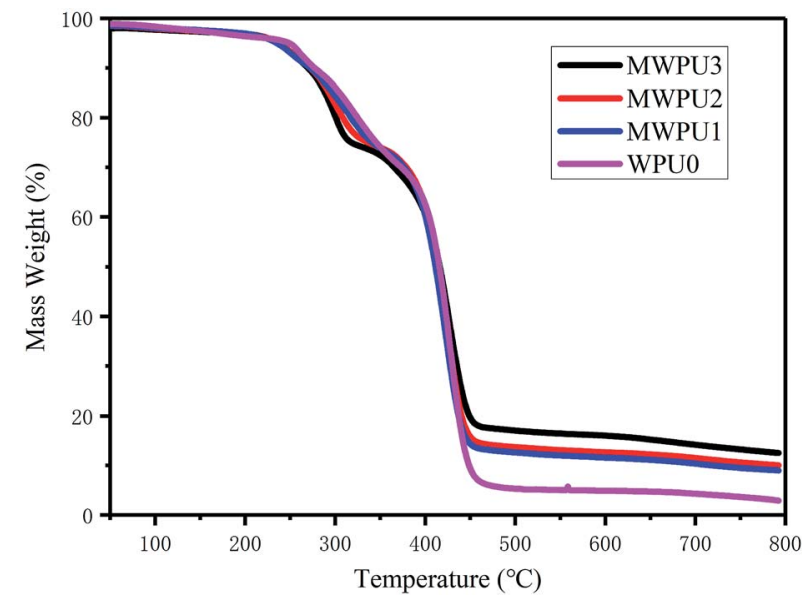

Fig. 4 TGA curves of synthesized WPUO and MWPUs.

Table 3 Thermal decomposition temperature corresponding to different numerical Thermogravimetry

\begin{tabular}{lccccc}
\hline Sample & $T_{10 \%}\left({ }^{\circ} \mathrm{C}\right)$ & $T_{20 \%}\left({ }^{\circ} \mathrm{C}\right)$ & $T_{30 \%}\left({ }^{\circ} \mathrm{C}\right)$ & $T_{40 \%}\left({ }^{\circ} \mathrm{C}\right)$ & $T_{50 \%}\left({ }^{\circ} \mathrm{C}\right)$ \\
\hline WPU0 & 269.66 & 300.76 & 365.44 & 400.23 & 415.73 \\
MWPU1 & 271.84 & 308.46 & 373.58 & 401.23 & 411.39 \\
MWPU2 & 272.18 & 317.47 & 376.58 & 402.72 & 414.06 \\
MWPU3 & 277.02 & 324.83 & 378.91 & 404.39 & 416.06
\end{tabular}

polyurethanes increases. For example, the $T_{10 \%}$ of WPU0 and MWPU3 were $269.66^{\circ} \mathrm{C}$ and $277.02{ }^{\circ} \mathrm{C}$. The $T_{30 \%}$ of WPU0 and MWPU3 were $365.44^{\circ} \mathrm{C}$ and $378.91^{\circ} \mathrm{C}$. So, the introduction of TA increased the thermal decomposition temperature of the waterborne polyurethanes. This was not only because of the introductive large amount of thermally-stable benzene ring, but also due to the secondary interactions between the ester bonds and heterocycles. Besides, it should be noted that the effect of TA was mainly on the first two thermal decomposition stages. Besides, residual polyurethanes at $700{ }^{\circ} \mathrm{C}$ enhanced as the content of introductive TA increased. ${ }^{23-25}$

\section{The influences of TA on the mechanical properties of the synthesized MWPUs}

The mechanical properties of the waterborne polyurethanes were analysed by the Young's modulus, tensile strength and elongation at break. The results were shown in Fig. 5 and Table 4. Young's modulus is a physical quantity that describes the deformation resistance of solid materials, which depends only on the physical properties of the material itself. The size of young's modulus indicates the rigidity of the material. The larger young's modulus is, the less likely it is to deform.

As the content of TA increases, the Young's modulus and tensile strength of the waterborne polyurethanes increased. Specifically, the Young's modulus of WPU0 and MWPU3 were 5.1 MPa and 12.4 MPa, respectively. Meanwhile, the tensile strength of WPU0 and MWPU3 were 8.5 MPa and 13.6 MPa, respectively. The increase of the Young's modulus and tensile strength of the waterborne polyurethanes after incorporation of
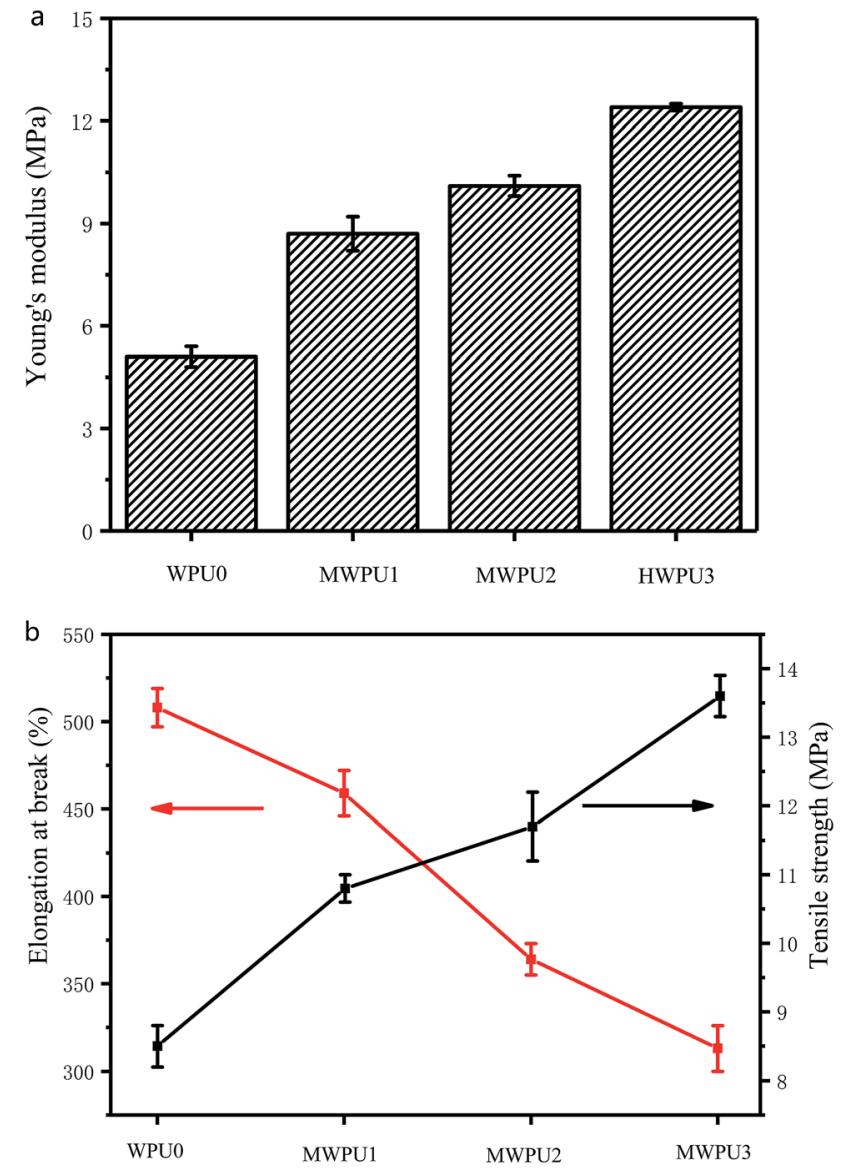

Fig. 5 Young's modulus (a), tensile strength and elongation at break (b) of synthesized WPUO and MWPUs.

TA is attributed to the fact that the introduction of TA not only enhances the structural rigidity of waterborne polyurethanes, but also increases the hydrogen bonds and the molecular interactions of waterborne polyurethanes, thus ultimately enhancing the mechanical strength of waterborne polyurethanes. However, it is worth noting that the increase of TA content in the synthesized MWPUs resulted in a decrease in the elongation at break of the waterborne polyurethanes (Table 4), which is due to the decrease of the flexibility of chains of MWPUs.

\section{The antioxidation capacity of the synthesized MWPUs}

DPPH radical scavenging assay. Typically, the antioxidation capacity of materials is mainly depending on their ability to donate electron to radical. To investigate the effects of

Table 4 Young's modulus, tensile strength and elongation at break of the synthesized WPUO and MWPUs

\begin{tabular}{lccl}
\hline Sample & $\begin{array}{l}\text { Young's } \\
\text { modulus (MPa) }\end{array}$ & $\begin{array}{l}\text { Tensile strength } \\
(\mathrm{MPa})\end{array}$ & $\begin{array}{l}\text { Elongation at } \\
\text { break }(\%)\end{array}$ \\
\hline WPU0 & $5.1 \pm 0.3$ & $8.5 \pm 0.3$ & $508 \pm 11$ \\
MWPU1 & $8.7 \pm 0.5$ & $10.8 \pm 0.2$ & $459 \pm 13$ \\
MWPU2 & $10.1 \pm 0.3$ & $11.7 \pm 0.5$ & $364 \pm 9$ \\
MWPU3 & $12.4 \pm 0.1$ & $13.6 \pm 0.3$ & $313 \pm 13$
\end{tabular}




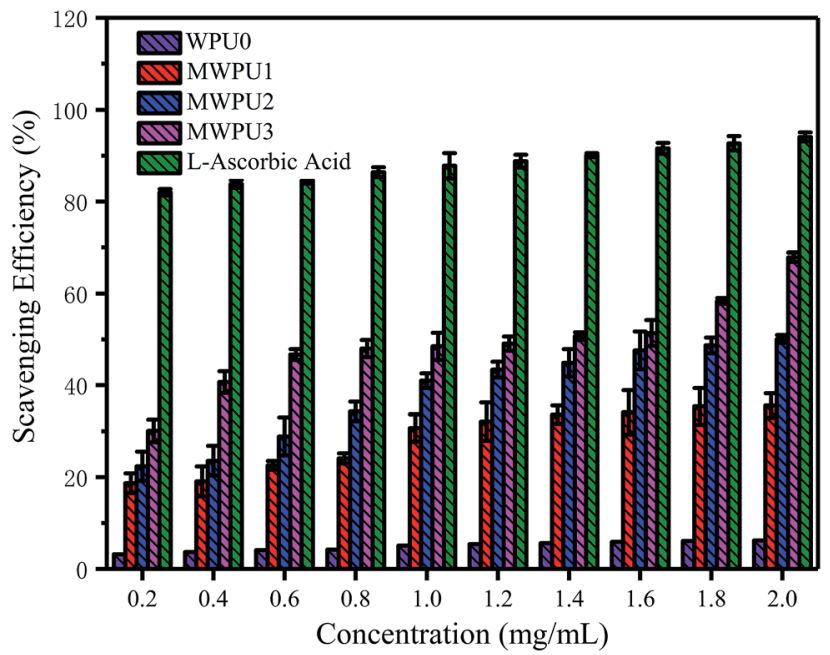

Fig. 6 DPPH radical scavenging efficiency of synthesized WPUO and MWPUs.

incorporation of TA in MWPUs on their antioxidation capacity, the DPPH radical scavenging activities of the synthesized WPUO and MWPUs were measured. As illustrated in Fig. 6, the WPU0 without TA did not exhibited any DPPH radical scavenging activity, whereas, the synthesized TA-based MWPUs showed outstanding radical scavenging activity. Besides, the radical scavenging activity of TA-based MWPUs enhanced as TA content increases. This result demonstrated that $\mathrm{TA}$ is the main component in MWPUs contributing to the antioxidant activity. In addition, the radical scavenging activity of MWPUs also get strengthen as the concentration of MWPUs increase. Particularly, when the solution concentration of MWPUs is $2 \mathrm{mg} \mathrm{ml} \mathrm{m}^{-1}$, the DPPH radical scavenging efficiency is up to $67 \%$. This excellent DPPH radical scavenging activity is mainly attributed to the catechol groups of TA that can serve as electron donors and react with free radicals effectively to convert them into more stable products. ${ }^{26,27}$

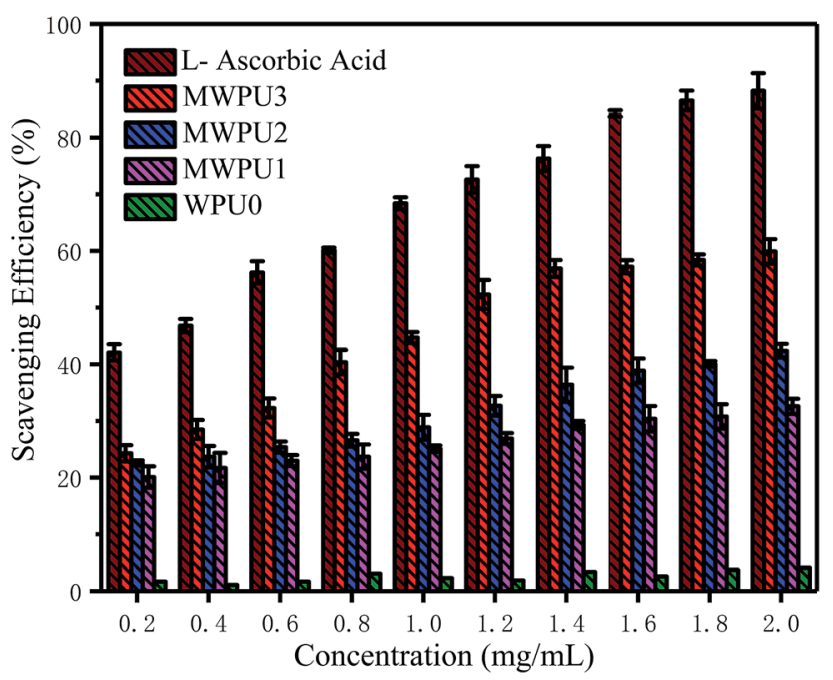

Fig. 7 Hydroxyl radical scavenging efficiency of synthesized WPUO and MWPUs.
Hydroxyl radical scavenging assay. Hydroxyl radical, as the most toxic radical among the reactive oxygen species (ROS) and one of the most active radicals, can react with a range of substances in human body, thus leading to a variety of diseases. Given that, hydroxyl radical scavenging activities of the synthesized WPU0 and MWPUs were further investigated. As presented in Fig. 7, WPU0 did not exhibit any scavenging activities for hydroxyl radical, and the hydroxyl radical scavenging activities of TA-based MWPUs increase with the increase of TA content and the concentration of MWPUs in solution. These results are generally consistent with the results of DPPH radical. Particularly, when the concentration of MWPU3 solution is $2 \mathrm{mg} \mathrm{ml}^{-1}$, the hydroxyl radical scavenging efficiency is over $50 \%$, further confirming the excellent antioxidation capacity of the TA-based MWPUs.

\section{The degradation behaviours of WPUO and MWPUs}

The degradation behaviours of WPU0 and MWPUs were studied by determining weight loss over time under two different conditions including in simulated body fluid and in soil. Fig. 8a represented the degradation curves of WPUO and MWPUs in
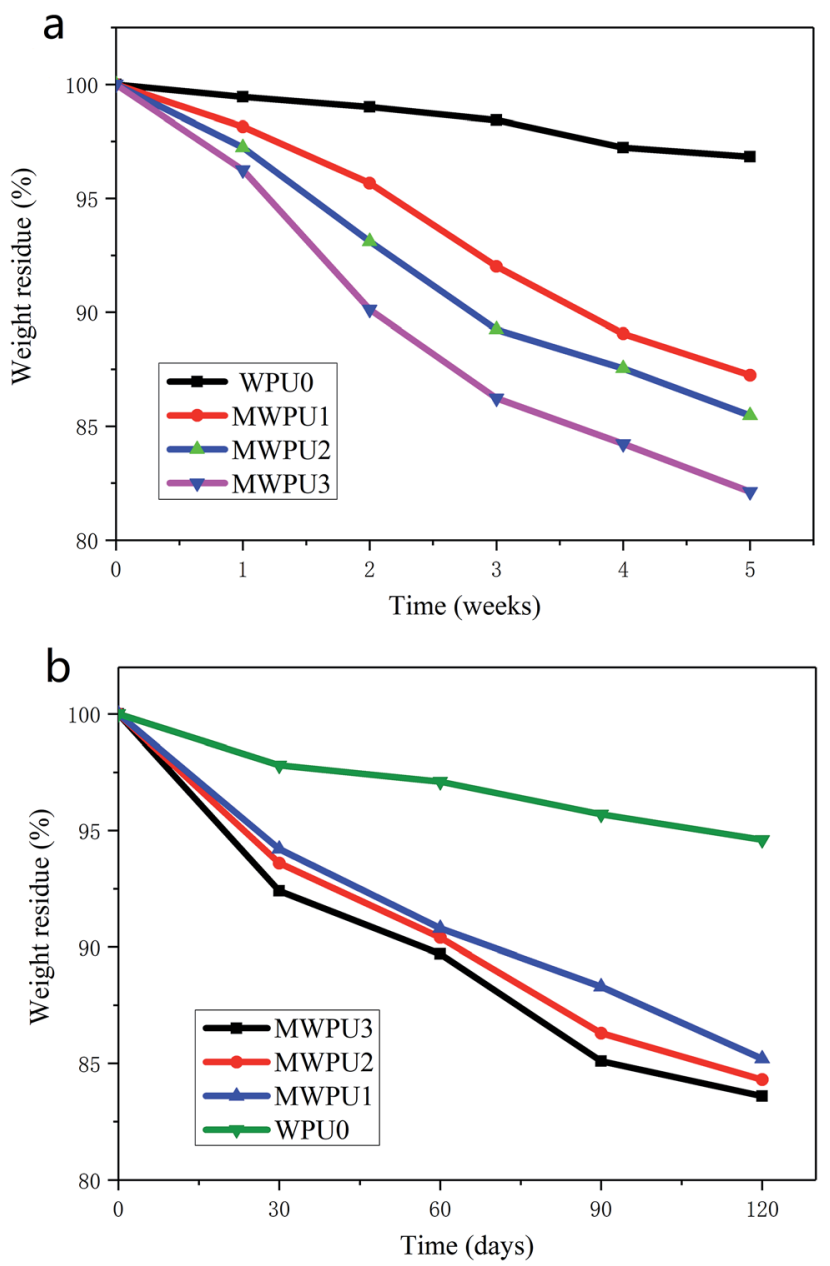

Fig. 8 Degradation curves of synthesized WPUO and MWPUs in simulated body fluid (a) and in soil (b). 


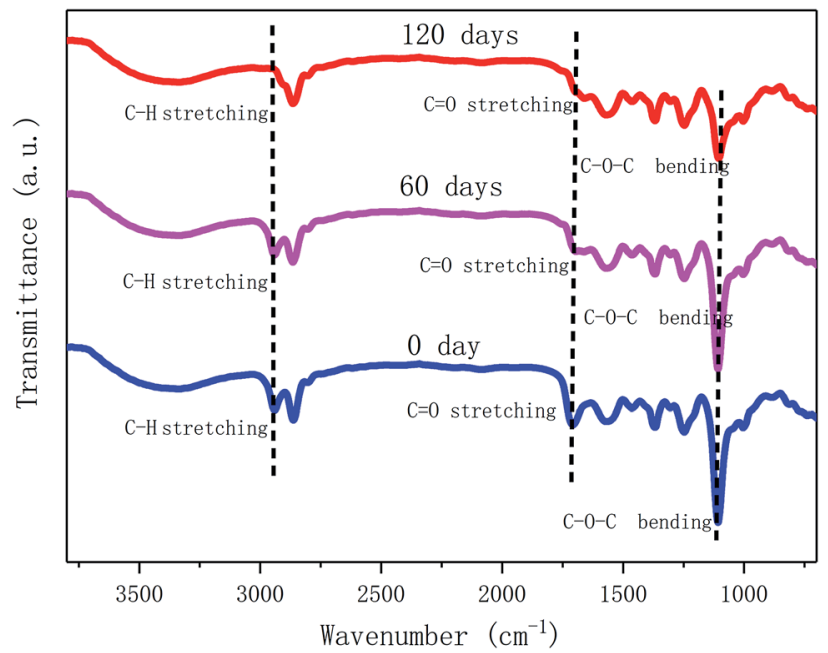

Fig. 9 FT-IR spectra of MWPUs before and after 60 days and 120 days degradation.

simulated body fluid (PBS solution). It is found that after 5 weeks of degradation in simulated body fluid, the TA-based MWPUs exhibited much high weight loss compared with WPU0, indicating that TA-based MWPUs have better degradation performances. And as the increase of TA content in MWPUs, the weight loss of TA-based MWPUs rising. For instance, the weight loss of MWPU3 after 5 weeks' degradation is up to $18 \%$, which is higher than the performance of MWPU1 and MWPU2. The outstanding degradation performance of
MWPU3 is due to the presence of large number of ester bonds in the chains of MWPU3 that is liable to hydrolysis.

The degradation behaviours of WPU0 and MWPUs in soil were also investigated and the results are shown in Fig. 8b. After 120 days degradation in soil, TA-based MWPUs exhibited 15$17 \%$ weight loss, which is much higher than $5 \%$ weight loss of WPU0, further confirming the better degradation performance of TA-based MWPUs. It is worth noting that although the weight loss of TA-based MWPUs also exhibited a same trend in soil with the increase of TA content in MWPUs, the increase of TAbased MWPUs in soil is much lower than that in simulated body fluid.

To further investigate the degradation process of MWPU3 in soil, the structure of MWPU3 and the surface morphology of MWPU3 films were characterized with FT-IR ATR and SEM, respectively. As shown in Fig. 9, significant changes of three peaks concentrate upon 2864-2940 $\mathrm{cm}^{-1}, 1710 \mathrm{~cm}^{-1}$ and $1130 \mathrm{~cm}^{-1}$ have been observed. Particularly, the peak at 2864$2940 \mathrm{~cm}^{-1}$ is attributed to the $-\mathrm{CH}_{2}$ group and the peak intensity exhibited an obvious decrease after 120 days of degradation, which is mainly due to the degradation of $\mathrm{C}-\mathrm{H}$ bond caused by the hydrocarbon-degrading bacteria. The second peak with significant change at $1710 \mathrm{~cm}^{-1}$, which is ascribed to the $-\mathrm{C}=\mathrm{O}$ group. The peak intensity exhibited an apparent decrease after 60 days of degradation and this peak disappeared completely after 120 days. The degradation of $-\mathrm{C}=$ $\mathrm{O}$ group is mainly caused by hydrolysis and biodegradation of bacteria. The peak attributed to $\mathrm{C}-\mathrm{O}-\mathrm{C}$ bond $\left(1130 \mathrm{~cm}^{-1}\right)$ did not exhibited significant change after 60 days, whereas the peak

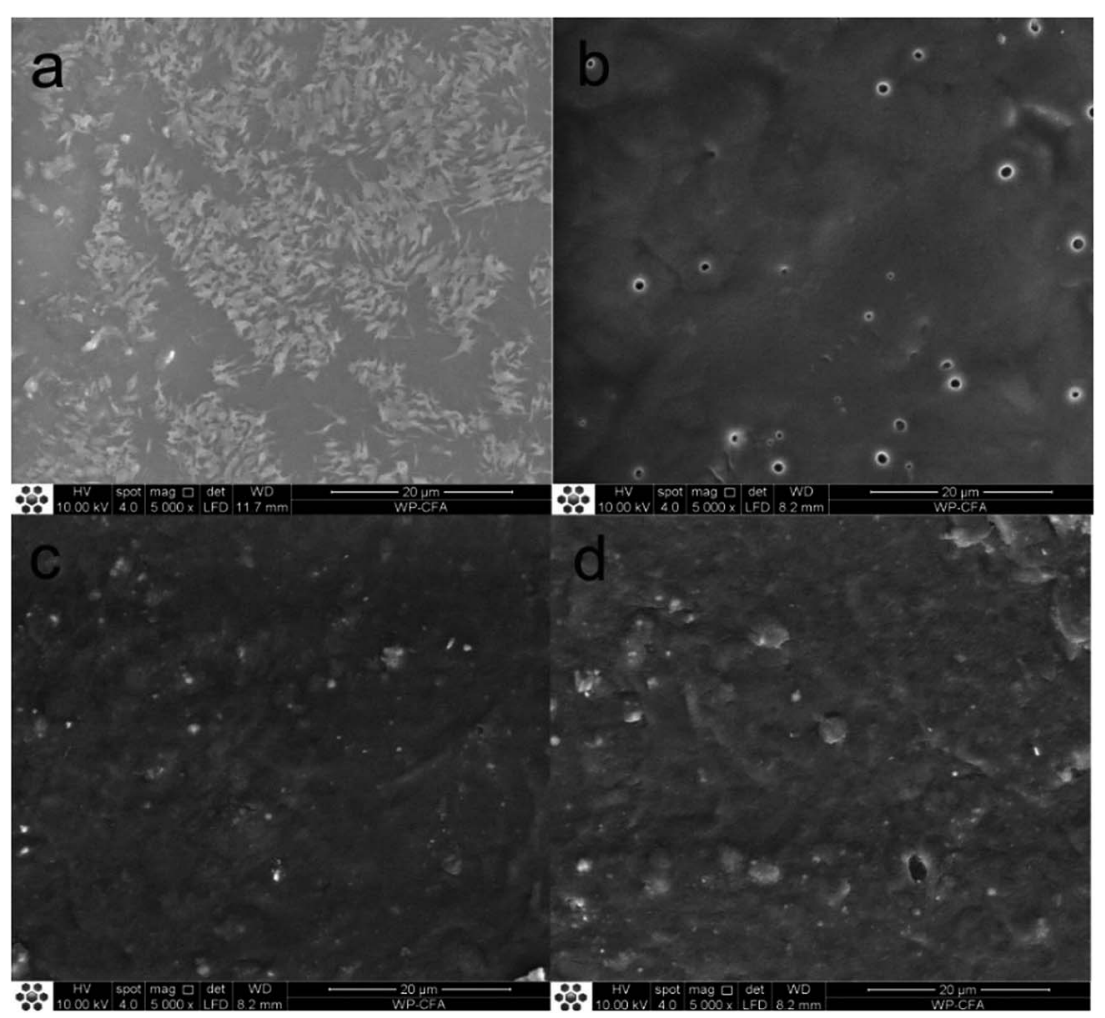

Fig. 10 Change of surface morphology of MWPU3 film before and after degradation. (a) 0 days, (b) 30 days, (c) 60 days and (d) 120 days. 
intensity showed a clear decrease after 120 days of degradation, which is probably due to the degradation of enzymes or bacteria in soil. That result also indicated that the degradation of $\mathrm{C}-\mathrm{O}-\mathrm{C}$ bonds mainly occurred in last stage. According to these findings, the degradation of TA-based MWPUs is mainly due to degradation of ester bonds, $\mathrm{C}-\mathrm{H}$ bonds and $\mathrm{C}-\mathrm{O}-\mathrm{C}$ bonds caused by the bacteria, enzymes and water existed in soil. ${ }^{28,29}$

The change of surface morphology of MWPU3 film during the degradation process was presented in Fig. 10. At the initial state, MWPU3 film exhibited a very smooth surface without any defect. However, as time goes, the surface of MWPU3 film become rough and some holes were observed on the film surface after 120 days of degradation, further confirmed the degradation of MWPU3.

\section{Conclusions}

In this study, biodegradable MWPUs were prepared by incorporation of TA in the structure of waterborne polyurethane. The UV-vis spectra and FT-IR spectra of the synthesized MWPUs confirmed the presence of TA. The result of DSC and TGA demonstrated that the incorporation of TA enhanced the thermal stability of MWPUs, and Young's modulus and tensile strength of the waterborne polyurethanes after incorporation of TA were increased due to the increase of structural rigidity, hydrogen bonds and molecular interactions of the MWPU chains. Besides, the synthesized TA-based MWPUs exhibited excellent antioxidation capacity and outstanding biodegradation property. Along with these excellent properties and sustainability of TA, the developed TA-based MWPUs exhibited great potential in a wide range of practical applications. MWPU is non-toxic, biodegradable and biocompatible, which is very promising in commercial applications. The degradation property of biodegradable materials as medical materials is concerned, as a long-term material implanted in the body, it is generally not expected to degrade. But, when used as similar tissue engineering scaffolds, medical adhesives and drug sustained-release materials, it is hoped that they can be gradually degraded in the body. Besides, MWPU can be used to make biodegradable condoms.

\section{Conflicts of interest}

There are no conflicts to declare.

\section{Acknowledgements}

This work was financially supported by the National Natural Science Foundation of China (81571801, 81671944), the Fundamental Research Funds for the Central Universities and the Open Funds for Characterization of Tongji University.

\section{References}

1 X. Ji, H. Wang and X. Ma, RSC Adv., 2017, 7(54), 34086-34095.
2 A. Santamaria-Echart, L. Ugarte and C. García-Astrain, Carbohydr. Polym., 2016, 151, 1203-1209.

3 Z. Zhong, S. Luo and K. Yang, RSC Adv., 2017, 7(67), 4229642304.

4 B. B. R. Silva, R. M. C. Santana and M. M. C. Forte, Int. J. Adhes. Adhes., 2010, 30(7), 559-565.

5 K. Liu, Z. Su and S. Miao, RSC Adv., 2016, 6(38), 31698-31704.

6 Z. Luo, Y. Shi and D. Zhao, Procedia Eng., 2011, 18(18), 37-42.

7 I. Phiwchai, W. Yuensook and N. Sawaengsiriphon, Eur. J. Pharm. Sci., 2017, 114, 64.

8 B. Ghosh, S. Gogoi, S. Thakur and N. Karak, Prog. Org. Coat., 2016, 90, 324-330.

9 X. Lai, Z. Hu and Y. Shen, Petrochem. Technol., 2017, 46(1), 83-89.

10 W. Y. Li, Y. M. Cao and X. Q. Zhou, Appl. Mech. Mater., 2012, 249-250, 842-848.

11 D. Villaño, M. S. Fernández-Pachón, M. L. Moyá, A. M. Troncoso and M. C. García-Parrilla, Talanta, 2007, 71(1), 230-235.

12 Q. Li, L. Qiu and W. Tan, RSC Adv., 2017, 7(67), 42225-42232. 13 X. G. Zhang, L. Tang and R. L. Shang, Storage Process, 2017, 17(6), 39-46.

14 N. Singh and P. S. Rajini, Food Chem., 2004, 85(4), 611-616. 15 L. Yu, S. Haley and J. Perret, J. Agric. Food Chem., 2002, 50(6), 1619-1624.

16 T. Kokila, P. S. Ramesh and D. Geetha, Appl. Nanosci., 2015, 5(8), 911-920.

17 H. Nur Maulida, F. Qulub and A. F. Rosdiani, J. Biomimetics, Biomater. Biomed. Eng., 2016, 28, 78-84.

18 J. L. Ryszkowska, M. Auguścik and A. Sheikh, Compos. Sci. Technol., 2010, 70(13), 1894-1908.

19 J. Liu, X. Qi and D. Zhang, Adv. Mater. Sci. Eng., 2017, 3, 1-14.

20 B. L. Dou, A. N. Qiu-Feng and M. A. Jun-Jian, Appl. Chem. Ind., 2010, 39, 189-191.

21 B. Ou, M. Chen and R. Huang, RSC Adv., 2016, 6(52), 4713847144.

22 J. Wang, P. Sun and Z. Zheng, Polym. Degrad. Stab., 2012, 97(11), 2294-2300.

23 G. Zheng, M. Lu and X. Rui, Appl. Surf. Sci., 2016, 399, 272281.

24 H. Fu, Y. Wang and X. Li, Compos. Sci. Technol., 2016, 126, 86-93.

25 X. Li, Y. Chen and S. Wu, Polym. Mater.: Sci. Eng., 2017, 33(7), 138-143.

26 E. Montanari, A. Gennari and M. Pelliccia, Macromol. Biosci., 2016, 16(12), 1815.

27 M. Shin, K. Kim and W. Shim, ACS Biomater. Sci. Eng., 2016, 2(4), 687-696.

28 L. Cosgrove, P. L. Mcgeechan, G. D. Robson and P. S. Handley, Appl. Environ. Microbiol., 2007, 73(18), 5817.

29 J. C. Peeler, S. Schedin-Weiss and M. Soula, J. Biol. Chem., 2017, 292(52), 21623-21630. 